\title{
Finite element modelling of ion convection by
}

\section{electrostatic forces}

\author{
Geoffrey Deliége ${ }^{(1)}$, François Henrotte ${ }^{(2)}$, Wim Deprez ${ }^{(3)}$ and Kay Hameyer ${ }^{(2)}$ \\ (1) K.U. Leuven, Dept. Computer Science (TWR), Celestijnenlaan 200A, B-3001 Heverlee, Belgium \\ (2) Institut für Elektrische Maschinen (RWTH), Schinkelstrasse 4, 52056 Aachen, Germany \\ (3) K.U. Leuven, Dept. ESAT-ELECTA, Kasteelpark Arenberg 10, B-3001 Heverlee, Belgium
}

\begin{abstract}
Different formulations of the problem of a cloud of ions convected by electrostatic forces are presented. Their influence on the ionic charge conservation is discussed. It is shown that a mixed electrostatic formulation is the most accurate for the studied example.
\end{abstract}

Index Terms-Finite element, electrostatic, source field, mixed formulation.

\section{INTRODUCTION}

$\mathbf{T}$ HE numerical analysis of an electrostatic painting process [1] requires to solve the classical electrostatic equations coupled with a charge conservation equation,

$$
\partial_{t} \rho_{i}+\nabla \cdot \underline{j}=0
$$

where $\rho_{i}$ is the ion charge density. The purpose of this paper is to determine whether the current density is preferably expressed as $\underline{j}=\frac{\mu}{\varepsilon} \underline{d} \rho_{i}$ or $\underline{j}=\mu \underline{e} \rho_{i}$. At the discrete level, the first expression ensures continuity of the normal component of $\underline{j}$, across material discontinuities and inter-element boundaries, which is the natural continuity for a flux density (here a flux of ionic charge, $\left.[j]=\left[\mathrm{Cm}^{-2} \mathrm{~s}^{-1}\right]\right)$. On the other hand, the second

This article is dedicated to Prof. G. Henneberger, who is released into his well deserved retirement with gratitude and appreciation for his meritorious scientific work.

This text presents research results of the Belgian programme on Interuniversity Poles of Attraction initiated by the Belgian State, Prime Minister's Office, Science Policy Programming. expression, which is more customary, ensures the continuity of the tangential component of $\underline{j}$. In particular, the influence on the conservation of the total charge in the system during the transient process is analysed.

\section{EQUATIONS}

The device consists of a set of thin wires parallel to a grounded iron plate. The wires are brought to a negative potential of high amplitude. The resulting electric field is particularly strong around the wires and causes the acceleration of free electrons which move away from the cathode and combine with atoms. The negative ions drift toward the anode, i.e. the grounded plate, due to Coulomb forces. The model is limited to a box extending from the middle of a wire to half the distance between two consecutive wires (Fig. 1).

In the absence of coating particles, the ion drift is described by the equations

$$
\begin{aligned}
\nabla \cdot \underline{d} & =\rho_{i}, \\
\nabla \times \underline{e} & =0, \\
\underline{d} & =\varepsilon_{0} \underline{e}, \\
\partial_{t} \rho_{i}+\nabla \cdot\left(\mu_{i} \underline{e} \rho_{i}\right) & =0 .
\end{aligned}
$$

where $\mu_{i}$ is the ion mobility [ $\left.\mathrm{Vs}^{-1}\right]$ [2].

The system of equations (2-5) defines an electrostatic problem coupled with a transient convection problem. They 


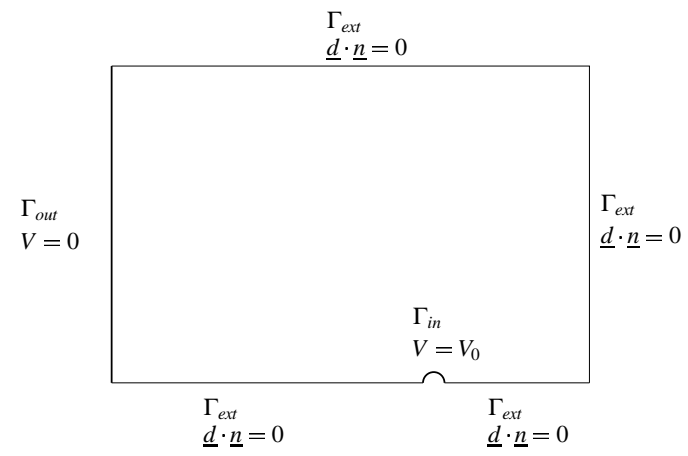

Fig. 1. Boundary conditions for the finite element model of the electrostatic painting device.

are solved separately: the transient equation is numerically integrated in time and the static problem is solved at each time step. Non-linear iterations can be avoided if the time step is small enough to ensure a slow evolution of the solution.

\section{ELECTROSTATIC FORMULATIONS}

\section{A. Electric scalar potential $V$}

The classical scalar potential formulation reads:

Find $V \in \mathrm{H}_{V}(\Omega)$ such that

$$
\int_{\Omega} \nabla V^{\prime} \cdot \nabla V d \Omega-\int_{\Omega} V^{\prime} \frac{\rho_{i}}{\varepsilon_{0}} d \Omega=0
$$

$\forall V^{\prime} \in \mathrm{H}_{V 0}(\Omega)$,

with the function spaces

$$
\begin{aligned}
\mathrm{H}_{V} & =\left\{V \in \mathrm{H}(\operatorname{grad}, \Omega):\left.V\right|_{\Gamma_{\text {in }}}=V_{0},\left.V\right|_{\Gamma_{\text {out }}}=V_{1}\right\} \\
\mathrm{H}_{V 0} & =\left\{V \in \mathrm{H}(\mathrm{grad}, \Omega):\left.V\right|_{\Gamma_{\text {in }}}=\left.V\right|_{\Gamma_{\text {out }}}=0\right\}
\end{aligned}
$$

\section{B. Electric vector potential $\underline{w}$}

The electric flux density is defined as

$$
\underline{d}=\underline{d}_{s}+\nabla \times \underline{w}
$$

where $\underline{d}_{s}$ is a source field such that $\nabla \cdot \underline{d}_{s}=\rho_{i}$ so that (2) is satisfied exactly. The weak formulation reads:

Find $\underline{d}_{s} \in \mathrm{H}_{d s}$ and $\underline{w} \in \mathrm{H}_{w}$ such that

$$
\int_{\Omega} \frac{1}{\varepsilon_{0}} \nabla \times \underline{w}^{\prime} \cdot\left(\underline{d}_{s}+\nabla \times \underline{w}\right) d \Omega=0
$$

$\forall \underline{w}^{\prime} \in \mathrm{H}_{w 0}$, with the function spaces

$$
\begin{aligned}
\mathrm{H}_{d s}= & \left\{\underline{d}_{s} \in \mathrm{H}(\operatorname{div}, \Omega):\right. \\
& \left.\underline{d}_{s} \mid \Gamma_{i n} \cup \Gamma_{\text {ext }}=0, \nabla \cdot \underline{d}_{s}=\rho_{i} \text { in } \Omega\right\}, \\
\mathrm{H}_{w}= & \{\underline{w} \in \mathrm{H}(\operatorname{curl}, \Omega): \\
& \left.\nabla \times \underline{w} \mid \Gamma_{e x t}=0, \int_{\partial \Gamma_{i n}} \underline{w}=\varphi_{d}\right\}, \\
\mathrm{H}_{w 0}= & \left\{\underline{w} \in \mathrm{H}(\operatorname{curl}, \Omega): \underline{w} \mid \Gamma_{e x t} \cup \partial \Gamma_{i n}=0\right\} .
\end{aligned}
$$

The source field is built prior to the assembly on basis of the current value of the ion charge density. It is discretised with face elements [3], which are in 2D associated with the edges of the mesh. The vector potential has a non-zero component in the Z-direction only and is therefore discretised with nodal elements, like the magnetic vector potential $\underline{a}$.

Since the potential $V$ cannot be fixed in this formulation, a constraint is set on the total flux of $\underline{d}$ through the boundary of the wire $\Gamma_{i n}$. This amounts to fix the circulation of $\underline{w}$ on the contour of $\Gamma_{\text {in }}$ because $\underline{d}_{s}$ is zero on this boundary.

\section{Mixed $\underline{d}-V$ formulation}

The unknown fields are the scalar potential $V$ and the electric flux density $\underline{d}$. A weak formulation of (2) and (4) is solved:

Find $\underline{d} \in \mathrm{H}_{d}$ and $V \in \mathrm{H}_{V}$ such that

$$
\begin{array}{r}
\int_{\Omega} \nabla V^{\prime} \cdot \underline{d} d \Omega+\int_{\Omega} V^{\prime} \rho_{i} d \Omega \\
-\quad \int_{\Gamma_{\text {in }} \cup \Gamma_{\text {out }}} V^{\prime} \underline{d} \cdot \underline{n} d \Gamma=0,
\end{array}
$$

$\forall V^{\prime} \in \mathrm{H}_{V 0}$, and

$$
\int_{\Omega} \frac{1}{\varepsilon_{0}} \underline{d}^{\prime} \cdot \underline{d} d \Omega+\int_{\Omega} \underline{d}^{\prime} \cdot \nabla V d \Omega=0
$$

$\forall \underline{d}^{\prime} \in \mathrm{H}_{d}$,

with the function space

$$
\mathrm{H}_{d}=\left\{\underline{d} \in \mathrm{H}(\operatorname{div}, \Omega):\left.\underline{d}\right|_{\Gamma_{e x t}}=0\right\},
$$

in addition to (7-8). Fields $V$ and $\underline{d}$ are discretised with nodal and face elements respectively. 
The resulting algebraic system takes the form

$$
\left[\begin{array}{cc}
A & B \\
B^{T} & 0
\end{array}\right]\left[\begin{array}{l}
x_{d} \\
x_{V}
\end{array}\right]=\left[\begin{array}{l}
0 \\
b
\end{array}\right],
$$

where $x_{d}$ and $x_{V}$ are the unknown dofs of the fields $\underline{d}$ and $V$. The empty block on the diagonal of the system (17) is characteristic of mixed formulations.

Babuška and Brezzi have proved that the discrete function spaces for the unknown fields of a mixed problem solved with finite elements must satisfy the so-called Babuška-Brezzi (BB) condition [4]. In fluid mechanics, this condition prevents some combinations of shape functions to be used for the velocity and pressure in incompressible Navier-Stokes equations: equal order elements, for example, lead to spurious pressure oscillations. One possibility is to discretise the pressure and the velocity with first order and second order elements respectively. In order to apply this result to our electrostatic formulation, we should discretise $\underline{d}$ with second order face elements and $V$ with first order nodal elements.

An alternative has been proposed by Hughes et al. who make use of modified weighting functions in order to circumvent the BB condition [5], [6]. The pressure-stabilised PetrovGalerkin (PSPG) method allows the velocity and pressure to be discretised with equal order elements. In fluid mechanics, the weighting functions for the momentum equations become

$$
\underline{v}^{\prime} \rightarrow \underline{v}^{\prime}+\tau_{e} \nabla p^{\prime}
$$

where $\underline{v}$ is the velocity, $p$ is the pressure and $\tau_{e}$ is an element-wise constant free parameter, comparable with the free parameter of the SUPG formulation, which is empirically determined. By analogy, the PSPG method can be applied to the mixed electrostatic formulation by modifying the weighting functions of (15),

$$
\underline{d}^{\prime} \rightarrow \underline{d}^{\prime}+\tau_{e} \nabla V^{\prime}
$$

which amounts to adding the following terms to the left-hand side:

$$
\tau_{e} \frac{1}{\varepsilon_{0}} \int_{\Omega} \nabla V^{\prime} \cdot \underline{d} d \Omega+\tau_{e} \int_{\Omega} \nabla V^{\prime} \cdot \nabla V d \Omega .
$$

The second advantage of the method is that the structure of the system (17) becomes

$$
\left[\begin{array}{cc}
A & B \\
\left(1+\tau_{\mathbf{e}}\right) B^{T} & \mathbf{C}
\end{array}\right]\left[\begin{array}{l}
x_{d} \\
x_{V}
\end{array}\right]=\left[\begin{array}{l}
0 \\
b
\end{array}\right],
$$

which is no more indefinite. The choice of an appropriate value of $\tau_{e}$ is discussed in section $\mathrm{V}$.

\section{TIME INTEGRATION SCHEMES}

A time integration scheme suited for convection equations must be chosen for (5). Two schemes based on Padé approximants are used, an explicit $\left(R_{0,3}\right)$ and an implicit one $\left(R_{2,1}\right)$.

\section{A. Explicit $R_{0,3}$}

The Taylor-Galerkin scheme derives from the approximant $R_{0,3}$. It is third order accurate and requires a less severe condition on the time step than the Lax-Wendroff scheme [7]. In the multi-step version of the scheme, only first order derivatives are present:

$$
\begin{aligned}
\rho^{n+\frac{1}{3}} & =\rho^{n}+\frac{1}{3} \Delta t \partial_{t} \rho^{n}, \\
\rho^{n+\frac{1}{2}} & =\rho^{n}+\frac{1}{2} \Delta t \partial_{t} \rho^{n+\frac{1}{3}}, \\
\rho^{n+1} & =\rho^{n}+\Delta t \partial_{t} \rho^{n+\frac{1}{2}} .
\end{aligned}
$$

\section{B. Implicit $R_{2,1}$}

This scheme is unconditionally stable for convection equations [8]. The two-steps expression avoids second order derivatives at the cost of an additional intermediate unknown, $\rho^{n+\frac{1}{2}}$ :

$$
\begin{aligned}
\rho^{n+\frac{1}{2}}+\frac{2}{3} \rho^{n+1}-\frac{1}{6} \Delta t \partial_{t} \rho^{n+1} & =0, \\
\rho^{n+1}+\Delta t \partial_{t} \rho^{n+\frac{1}{2}} & =\rho^{n}+\frac{1}{3} \Delta t \partial_{t} \rho^{n} .
\end{aligned}
$$




\section{RESUlts}

The different formulations are first studied on a simple testcase where the time step and the element size can easily be varied. The purpose is on the one hand to determine an optimal value for the free parameter in the stabilised mixed formulation, and on the other hand to study the convergence of charge conservation error for the electrostatic formulations. The error is defined as the residual of (5),

$$
R_{\rho}^{n}=\int_{\Omega} \rho_{i}^{n+1}-\rho_{i}^{n} d \Omega+\Delta t \int_{\partial \Omega} \underline{j} \cdot \underline{n} d \partial \Omega
$$

which quantifies the difference between the charge which has been gained or lost by the system through its boundaries during the time interval $\Delta t$, and the effective variation of the total charge. The relative error $r_{\rho}^{n}$ is the residual $R_{\rho}^{n}$ divided by the total charge at steady state. It can be integrated in time to characterise the global error for the whole integration process,

$$
r_{Q}=\sum_{n=1}^{n_{\max }}\left|r_{\rho}^{n}\right| .
$$

The real model is then solved to confirm the results obtained with the simple configuration.

\section{A. Test model}

The test model consists of a rectangular domain. The electric potential is fixed to 0 and $V_{\max }$ on the left boundary $\left(\Gamma_{\text {out }}\right)$ and the right boundary $\left(\Gamma_{\text {in }}\right)$ respectively. The initial distribution of $\rho_{i}$ is exponential in order to induce an intense electric field near the right boundary, as it occurs around the wire in the real problem.

In order to determine an optimal expression of $\tau_{e}$ for the stabilised $\underline{d}-V$ formulation, the electrostatic equations is solved on several meshes with a decreasing element size $h_{e}$. The number of BiCG steps as a function of $\tau_{e}$ is plotted in Fig. 2 for different values of $h_{e}$. The optimal value of $\tau_{e}$ lies for each mesh between 0 and -1 and does not strongly vary with $h_{e}$. Therefore, a constant value $\tau_{e}=-0.8$. all further computations have been made with

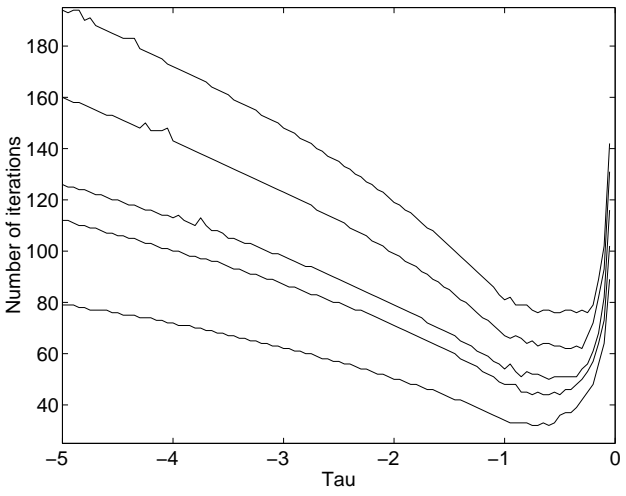

Fig. 2. Test model: Number of iterations of the BiCG solver for the stabilised mixed $\underline{d}-V$ formulation as a function of the parameter $\tau_{e}$, for different values of the element size $h_{e}$.

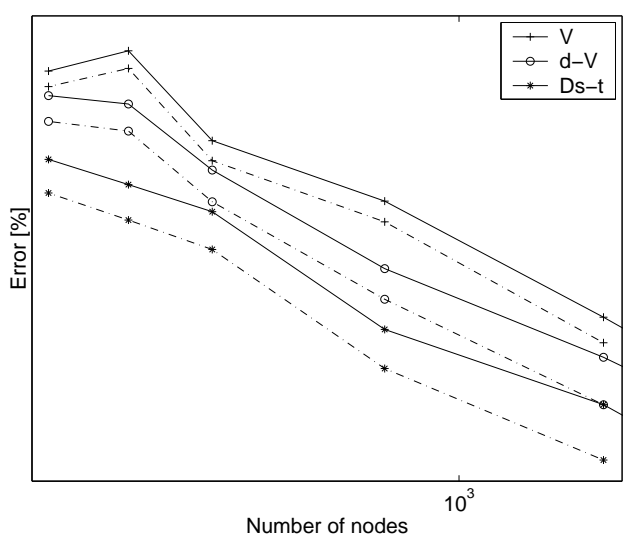

Fig. 3. Test model: Relative error $r_{Q}$ vs. number of nodes for the testcase solved with the different electrostatic formulations, with $\Delta t=510^{-6} \mathrm{~s}$ (continuous lines) and $\Delta t=2010^{-6} \mathrm{~s}$ (dotted lines).

The problem is solved with both explicit and implicit schemes for $\Delta t=510^{-6} \mathrm{~s}$, and with the implicit scheme for $\Delta t=2010^{-6} \mathrm{~s}$. The error $r_{Q}$ is plotted in Fig. 3. The implicit and explicit time schemes give similar results and the corresponding curves are identical.

It appears that the error is higher when $\underline{j}$ is expressed as $\underline{j}=\mu \underline{e} \rho_{i}$ (scalar potential formulation) than when expressed as $\underline{j}=\frac{\mu}{\varepsilon} \underline{d} \rho_{i}$, (vector potential and mixed formulations). The lowest error is obtained with the vector potential formulation with source field. 


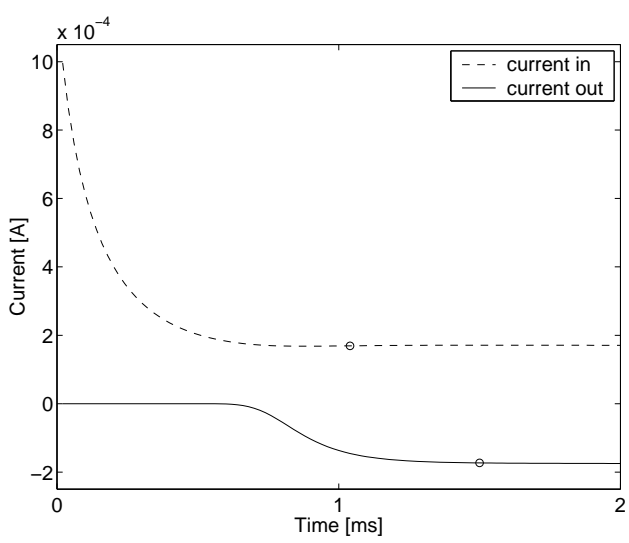

Fig. 4. Real model: Ion current flowing through the wire ('in') and the plate ('out'); the 'o' marks the point where the current has reached $99 \%$ of its steady state value.

\section{B. Real model}

A uniform charge density $\rho_{i}=10^{-9} \mathrm{C} / \mathrm{m}^{3}$ is assumed at $t=0$. First, ions are created around the wire and start moving away from it in all directions. At some distance of the wire, the charges are attracted by the plate where they are neutralised. The ionization phenomenon reaches a steady state after $1 \mathrm{~ms}$ (Fig. 4) and after $1.5 \mathrm{~ms}$, the flux of ions reaching the plate compensates the flux of ions leaving the wire to within $1 \%$. From that moment on, the total charge of the system must be constant.

The implicit time integration scheme is used with $\Delta t=$ $10^{-5}$ s. The explicit scheme is not used because its stability condition $C^{2}<1$, where $C$ is the CFL number [8], imposes such a restriction on the time step that tens of thousands of iterations would be necessary to reach steady-state.

The relative error $r_{\rho}^{n}$ is plotted in Fig. 7 as a function of time for the different formulations. It is maximum at the beginning of the computation, when the input current is maximum, and converges towards a constant. The error is the highest for the scalar potential formulation with first order elements. It decreases when second order elements are used but remains significantly higher than the error obtained with the $\underline{d}-V$ and $\underline{d}_{S}-\underline{w}$ formulations. The total error $r_{Q}$ is given in Table I

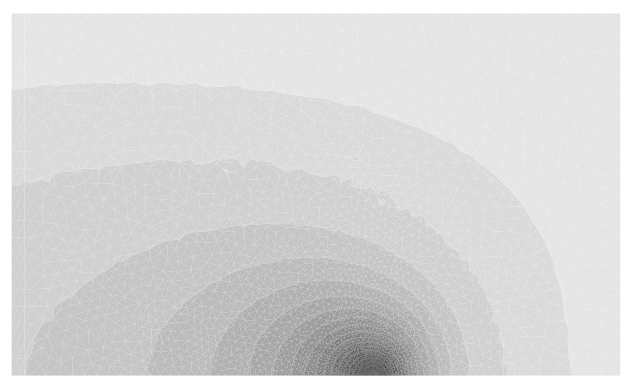

Fig. 5. Ion density $\rho_{i}$ at steady state $(t=2 \mathrm{~ms})$, ranging from $-3 \cdot 810^{-4} \mathrm{C} / \mathrm{m}^{3}$ (black) to $0 \mathrm{C} / \mathrm{m}^{3}$ (white).

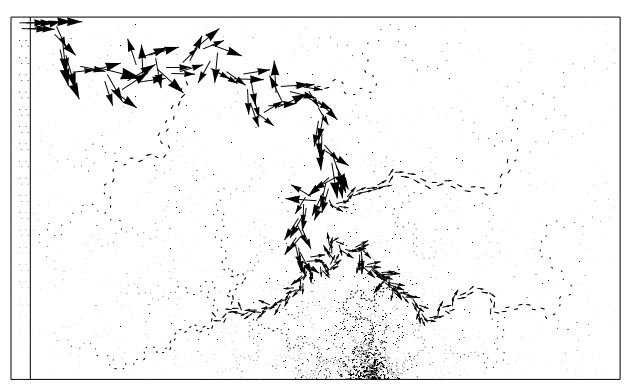

Fig. 6. Source field $\underline{d}_{s}$ at steady-state.

with the number of dofs of each formulation. The formulation with source field $\left(\underline{d}_{s}-\underline{w}\right)$ is apparently the most interesting, since it is the most accurate and requires only $n_{\text {node }}$ unknowns. However, the flux of $\underline{d}$ that is fixed by the boundary conditions is for this problem an unknown quantity which has to be determined in some way. In this case, the problem is first solved with a scalar potential formulation and $\varphi_{d}$ is calculated

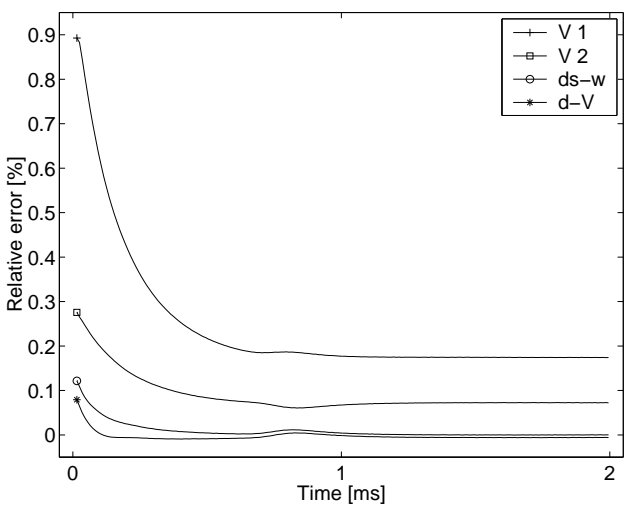

Fig. 7. Real model: relative error $r_{\rho}^{n}$ vs time; implicit scheme, 200 steps. 
TABLE I

COMPARISON OF THE RELATIVE ERRORS $r_{Q}$.

\begin{tabular}{c|c|c}
\hline Formulation & $r_{Q}[\%]$ & Nb dofs \\
\hline$V$, order 1 & 47.7 & $n_{\text {node }}$ \\
$V$, order 2 & 17.7 & $n_{\text {node }}+n_{\text {edge }}$ \\
$\underline{d}_{s}-\underline{w}$ & 1.8 & $n_{\text {node }}$ \\
$\underline{d}-V$ & 1.4 & $n_{\text {node }}+n_{\text {edge }}$ \\
\hline
\end{tabular}

as the integral of $-\varepsilon_{0} \nabla V$ on the boundary. On the contrary, the mixed formulation requires more unknowns but the boundary conditions on the potential are easily taken into account, and it is more accurate than the scalar potential formulation with second order elements.

\section{CONCLUSION}

The equations describing the drift of ions in an electrostatic painting device have been presented. Two unusual electrostatic formulations have been proposed in addition to the classical scalar potential formulation: the vector potential formulation with source field $\left(\underline{d}_{s}-\underline{w}\right)$ and the mixed formulation $(\underline{d}-V)$. A stabilisation technique for mixed problems, originally developed for Stokes problems and known as pressure stabilised Petrov-Galerkin (PSPG) formulation, has been successfully applied to the mixed electrostatic formulation. It has been shown that the formulations ensuring the continuity of the normal component of $\underline{j}$, i.e. the vector potential and mixed formulations, lead to a better charge conservation than the scalar potential formulation, even if $V$ is discretised with second order elements. The mixed formulation has on the vector potential formulation the advantage that the boundary conditions on $V$ can be easily expressed.

\section{REFERENCES}

[1] G. Deliége, F. Henrotte, and K. Hameyer. Finite element modelling of an electrostatic painting device. IEEE Transactions on Magnetics, 39(3):1432-1435, 2003.
[2] C. Gary and M. Moreau. L'effet couronne en tension alternative. Eyrolles, 1976.

[3] A. Bossavit. Computational Electromagnetism. Academic Press, Maryland, 1998.

[4] I. Babuška. The finite element method with Lagrangian multipliers. Numerische Mathematik, 20:179-192, 1973.

[5] L.P. Franca and T.J.R. Hughes. A new finite element formulation for computational fluid dynamics: VII. The Stokes problem with various wellposed boundary conditions: symmetric formulations that converge for all velocity/pressure spaces. Computer Methods in Applied Mechanics and Engineering, 65:85-96, 1987.

[6] T. De Mulder. Stabilized finite element methods for turbulent incompressible single-phase and dispersed two-phase flows. $\mathrm{PhD}$ thesis, Katholieke Universiteit Leuven, May 1997.

[7] J. Donea. A Taylor-Galerkin method for convective transport problems. International Journal for Numerical Methods in Engineering, 20:101$119,1984$.

[8] J. Donea, L. Quartapelle, and V. Selmin. An analysis of time discretisation in the finite element solution of hyperbolic problems. Journal of Computational Physics, 70:463-499, 1987. 